\title{
Decreased noise figure measurement uncertainty in $Y$ factor method
}

\author{
I. Gaspard \\ Hochschule Darmstadt, FB Elektrotechnik und Informationstechnik, Darmstadt, Germany \\ Correspondence to: I. Gaspard (ingo.gaspard@h-da.de)
}

\begin{abstract}
Almost always noise figure is measured today by using a matched noise source delivering two different but known noise temperatures ( $Y$ factor method). In commercially available noise sources these temperatures are characterized by the excess noise ratio (ENR) value, describing the ratio of equivalent noise power when switched on related to the noise power of a resistor at a temperature of $290 \mathrm{~K}$ (switched off). For a typical ENR value of $5 \mathrm{~dB}$ that means a hot temperature $T_{\mathrm{h}}=1207 \mathrm{~K}$ and a cold temperature $T_{\mathrm{c}}=290 \mathrm{~K}$.
\end{abstract}

\section{Introduction}

In this paper the impact of $T_{\mathrm{c}}$ onto the noise figure uncertainty is analysed. There are several advantages when holding $T_{\mathrm{c}}$ as low as possible and not - as usual done - at $290 \mathrm{~K}$. To realize $T_{\mathrm{c}}$ below room temperatures there are several possibilities, like cooling a resistor with liquid nitrogen or using sky noise by a suited antenna, see e.g. Zhutyaew (2010). Drawbacks of these solutions are either that they are expensive/need huge effort or are narrowband.

Therefore in this paper an alternative solution of a broadband noise generator on the basis of a Schottky diode operated in the conduction region will be described. In conduction region the Schottky diode shows a mixture of shot noise as well as thermal noise caused by the metal-semiconductor junction and the bulk resistance. By simple DC (direct current) measurements the overall noise temperature can be precisely determined and is in the range of $150 \mathrm{~K} \ldots 200 \mathrm{~K}$, depending on the diode type used. At the same time the bulk resistance in series with the differential resistance of the proper biased diode shows very good match to $50 \mathrm{Ohms}$ over a wide frequency range. Thus a noise normal can be implemented by simple DC and reflection factor measurement. Application and measurement results will be presented.

\section{Noise figure measurement uncertainty}

In the standard $Y$ factor measurement method to measure noise figure two main error sources are specified by equipment manufacturers: the uncertainty of the noise source in terms of $\triangle E N R$ and the uncertainty of the power ratio or instrumentation error $\Delta Y$. Typical values range e.g. between $\triangle \mathrm{ENR}= \pm 0.3 \mathrm{~dB}$ for an old Ailtech 7615 or $\Delta \mathrm{ENR}= \pm 0.2 \mathrm{~dB}$ for an HP346A noise source. For the instrumentation error of an HP8970A/B, Swain and Cox (1983), an instrumentation error of $\Delta Y= \pm 0.1 \mathrm{~dB}$ is specified. When analyzing the resulting error in noise figure (NF uncertainty, see annex) it turns out that the resulting NF uncertainty is strongly dependent on the cold temperature $T_{\mathrm{c}}$ of the noise source. In Fig. 1 the NF uncertainty is shown as a function of cold temperature of the source for two different ENR values of the source and for two LNAs which are assumed for simulation purpose to differ in noise figure $(\mathrm{NF}=0.1 \mathrm{~dB}$ and $\mathrm{NF}=0.5 \mathrm{~dB})$. From Fig. 1 it can be concluded that the cold temperature should be as low as possible to keep the resulting NF uncertainty as low as possible. It can also be seen that even for very low ENR $(\mathrm{ENR}=-4.5 \mathrm{~dB})$ the NF uncertainty is reasonable low if cold temperature is as low as e.g. $150 \mathrm{~K}$. Normally cold temperature of the noise source is at ambient room temperature, e.g. $290 \dot{\mathrm{K}}$. So it has

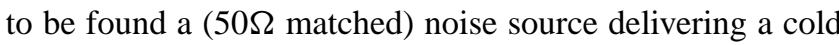
noise temperature as low as possible.

\section{Schottky diode}

Schottky diodes are formed by a metal-semiconductor junction and are widely used e.g. in mixers and detectors up to very high frequencies $(>100 \mathrm{GHz})$. This section will treat the necessary circuit models of a Schottky diode in order to provide a basic understanding of the noise generator implementation described in Sect. 4. 


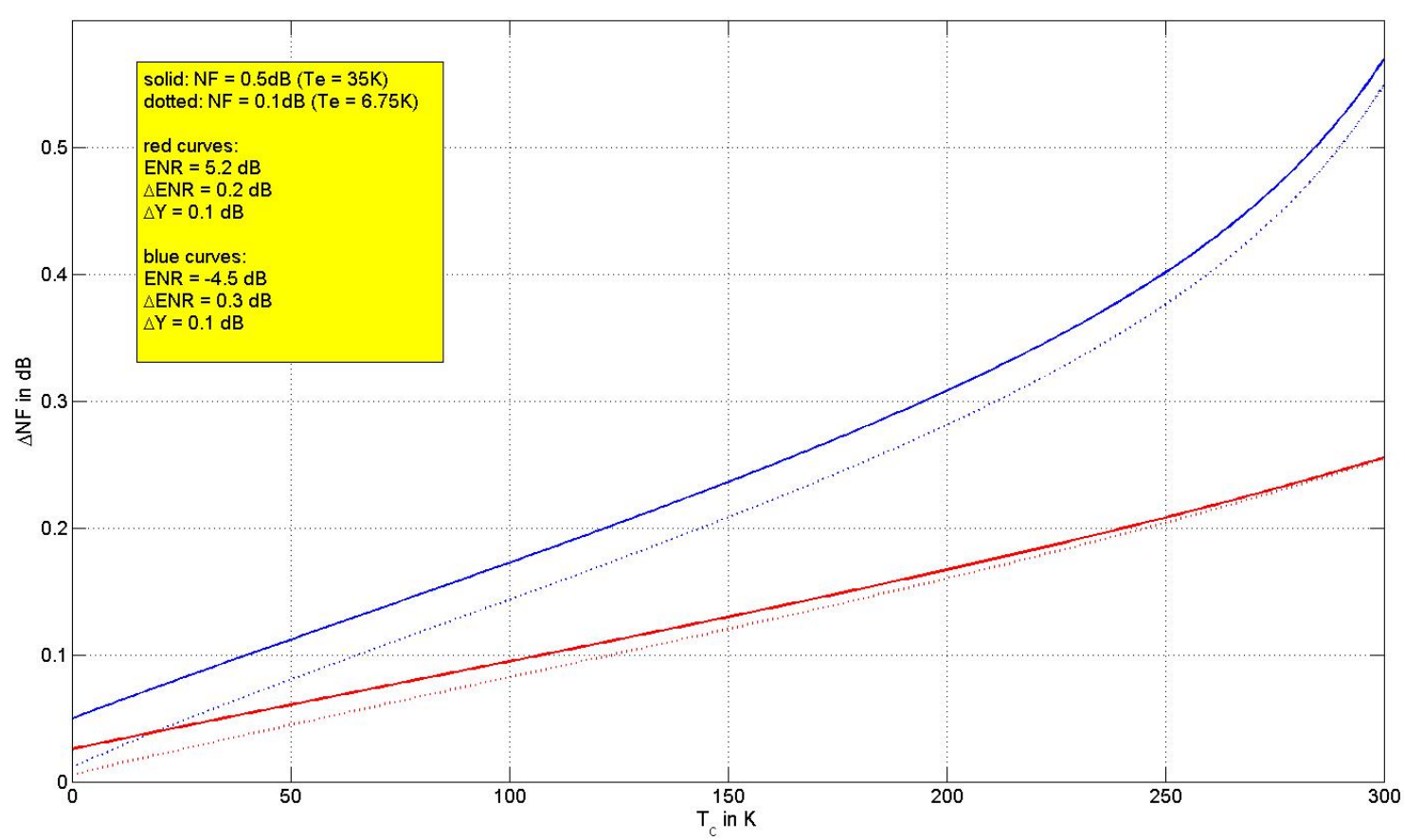

Fig. 1. Noise figure uncertainty in $\mathrm{dB}$ as function of $T_{\mathrm{c}}, \mathrm{NF}$ of DUT and ENR for $\triangle \mathrm{ENR}=0.2 \mathrm{~dB}$ (ENR calibration uncertainty of noise source) and $\Delta Y=0.1 \mathrm{~dB}$ (instrumentation uncertainty, e.g. HP8970).

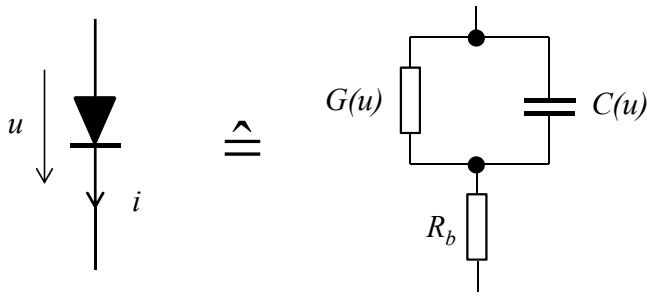

Fig. 2. Circuit model of Schottky diode.

\subsection{DC and small signal properties}

A widely used circuit model of the Schottky diode that is valid for both large-signal and small-signal analysis is given in Fig. 2, Maas (1993). It consists of a nonlinear resistance whose value is determined by the operational point and a capacitance representing the junction. Furthermore it contains a bulk resistance of a few Ohms. This model corresponds to the exponential $I / U$ characteristic expressed in the following equation

$i(u)=I_{\mathrm{S}} \cdot\left(\exp \left(\frac{q \cdot u}{\tilde{n} \cdot k T}\right)-1\right)$

where $k T / q$ describes the temperature voltage $(26 \mathrm{mV}$ at room temperature), $I_{\mathrm{S}}$ is the saturation current $(<0.1 \mu \mathrm{A})$ and $\tilde{n}$ is the so-called ideality factor, an empirical factor describing the deviation from the exponential (values range from 1 to 1.2).
$G(u)$ : nonlinear conductance of junction

$C(u)$ : nonlinear capacitance of junction

(very small, e.g. $<0.5 \mathrm{pF} \rightarrow$ high $\mathrm{f}$ )

$R_{b}$ : bulk resistance (e.g. $<10 \Omega$ )
By inspecting the DC $I / U$-characteristic of the Schottky diode (either by taking measurements or by looking to the data sheet) all necessary values to parameterize a suitable model of the Schottky diode can be derived - see Fig 3. That is namely the ideality factor $\tilde{n}$, the bulk resistance $R_{\mathrm{b}}$ and the saturation current $I_{\mathrm{S}}$.

\subsection{Noise model}

When operated in forward direction a Schottky diode shows a mixture of thermal noise due to the bulk resistance and shot noise due to the quantized charge carriers which have to climb the metal-semiconductor junction. That is summarized into a noise equivalent circuit with two independent noise sources given in Fig. 4 from Schiek et al. (2006). The conductance $G$ is the differential conductance of the diode at its DC operating point.

It turns out that the ratio of effective noise temperature at the diode's terminals to the ambient (physical) room 


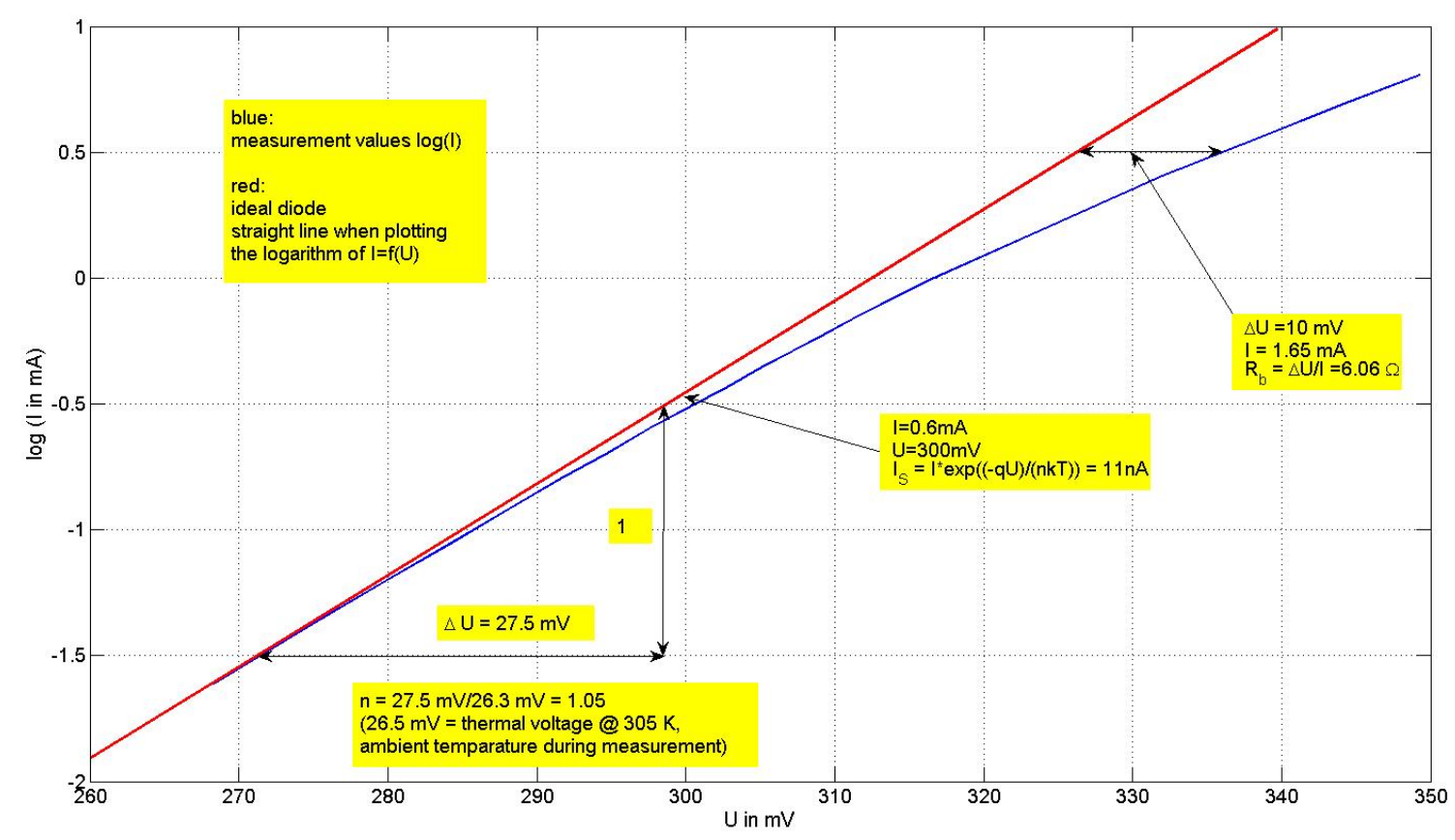

Fig. 3. $I / U$ characteristic of an Agilent HSMS-2823 Schottky diode.

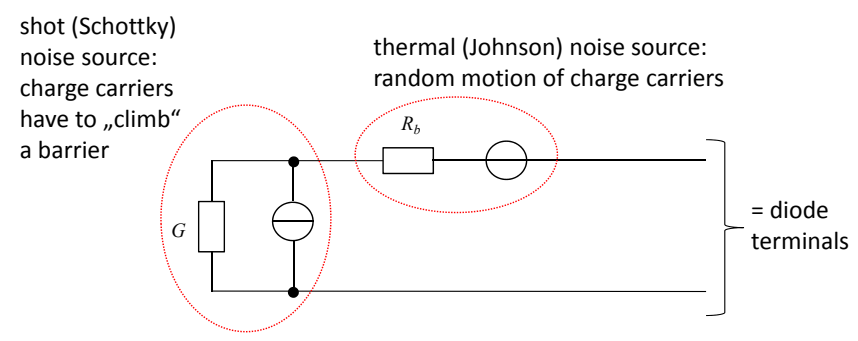

Fig. 4. Noise equivalent circuit model of a Schottky diode with bulk resistance.

temperature as a function of ideality factor $\tilde{n}$, DC current $I_{0}$ and saturation current $I_{\mathrm{S}}$ when neglecting the bulk resistance is given to be Schiek et al. (2006)

$\frac{T_{\mathrm{ef}}^{*}}{T}=\frac{1}{2} \cdot \tilde{n} \cdot\left(1+\frac{I_{\mathrm{S}}}{I_{0}+I_{\mathrm{S}}}\right)$

Inspecting Eq. (2) it can be seen that because the saturation current is negligible in comparison to the forward current $I_{0}$ the noise temperature could be at minimum half of the ambient (physical) temperature.

When including thermal noise from the bulk resistance and taking into account Eq. (2) the overall noise temperature $T_{\text {ef }}$ related to the ambient room temperature $T$ is then, see Schiek et al. (2006),

$\frac{T_{\mathrm{ef}}}{T}=\frac{\frac{T_{\mathrm{ef}}^{*}}{T}+R_{\mathrm{b}} \cdot G}{1+R_{\mathrm{b}} \cdot G}$

\section{Implementation}

Based on the diode model introduced in Sect. 3 a noise source by means of a Schottky diode operated in forward direction was implemented, see Figs. 5 and 6.

DC current through the diode can be aligned by the variable $10 \mathrm{k} \Omega$ resistor. A precision shunt of $1 \mathrm{k} \Omega$ allows DC current measurement. DC voltage across the diode's terminals can be measured directly at the N-male output connector. An additional PT1000 temperature sensor in the same housing placed nearby the diode allows measurement of the ambient temperature of the diode. RF (radio frequency) noise of the diode is decoupled from the DC part by a $10 \mathrm{k} \Omega$ chip resistor and $1 \mathrm{nF}$ disk capacitor.

By taking DC measurements in this setup bulk resistance, ideality factor and saturation current were derived according to Fig. 3 and are in good agreement to the data sheet.

\section{Application and measurements}

In order to provide a matched load the DC current through the diode was aligned to make the bulk resistance in series with the differential junction resistance a pure $50 \Omega$ load by means of VNWA (vector network analyzer) measurement. Thus $1 / G+R_{\mathrm{b}}$ is equal to $50 \Omega$. Best result was reached for DC current $I_{0}=0.67 \mathrm{~mA}$ at ambient temperature of $305 \mathrm{~K}\left(32^{\circ} \mathrm{C}\right)$. In Fig. 7 the measured return loss up to $1300 \mathrm{MHz}$ is shown. The degradation of return loss for increasing frequency is mainly caused by the junction capacitance $(\sim 0.7 \mathrm{pF})$ of the diode. More expensive diodes 


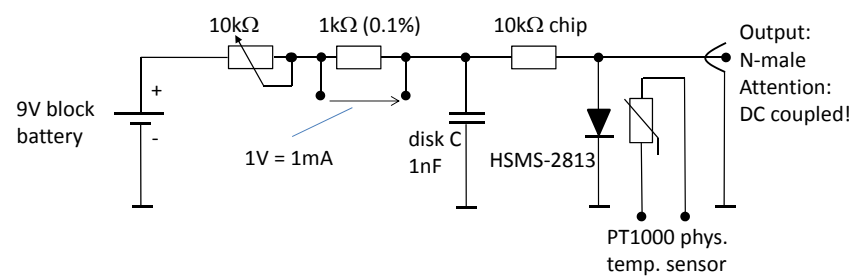

Fig. 5. Cicuit of implemented Schottky diode noise generator.

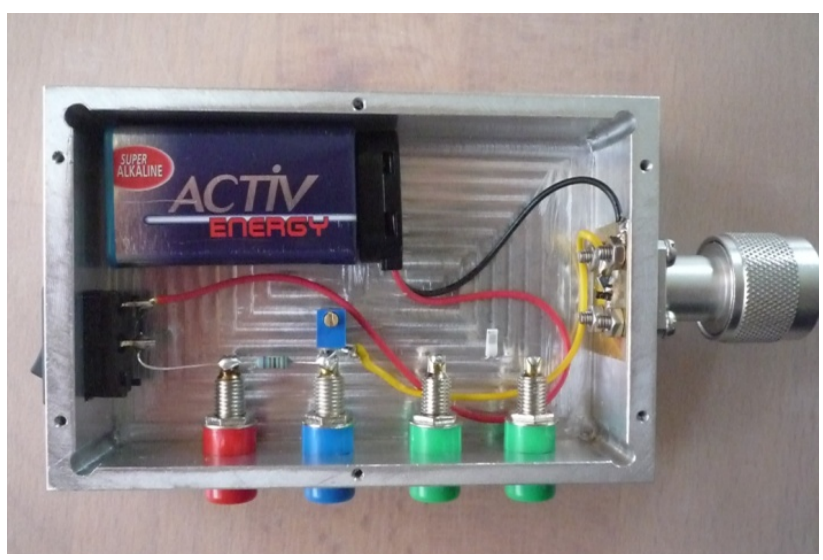

Fig. 6. Realization of noise generator.

with smaller junction capacitance should work at even much higher frequencies with sufficient return loss (RL). Of course the VNWA measurement has to be done carefully with low stimulus power (e.g. $<-20 \mathrm{dBm}$ ) in order to avoid nonlinear behavior of the diode.

Applying the noise model and calculus in Sect. 3 delivered an equivalent noise temperature of $180 \mathrm{~K}$ at $I_{0}=0.67 \mathrm{~mA}$ and ambient (physical) temperature of $305 \mathrm{~K}$ around the diode.

In Fig. 8 the combination of the Schottky noise generator with a standard noise generator is suggested in order to lower the overall cold temperature of the new noise source. This combination can be easily integrated into existing noise figure analyzer setups, e.g. the often used HP8970A/B. By combining the noise powers of the two noise generators by a directional coupler which has to have low loss in main line to avoid too much increasing of the overall cold temperature another advantage results: because of the low coupling $(-19.5 \mathrm{~dB})$ there is virtually no change in reflection coefficient for "on" and "off" state which would cause additional errors for mismatched devices under test ("gain error", see Bertelsmeier, 1988).

With the setup shown in Fig. 8 noise figure measurement at an MCL ZEL-1217LN low noise L-band amplifier was taken with an HP8970B noise figure analyzer. The noise figure was measured to be approximately $1 \mathrm{~dB}$ at $1296 \mathrm{MHz}$ which is in good agreement with data sheet (typically $1.1 \mathrm{~dB}$ ) and mea-

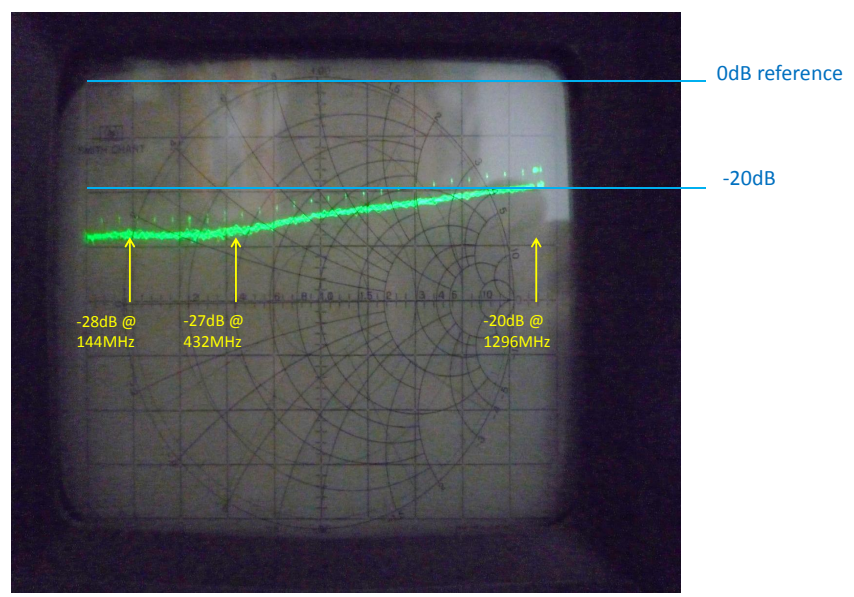

Fig. 7. Return loss (RL) of Schottky noise generator when biased for minimum RL.

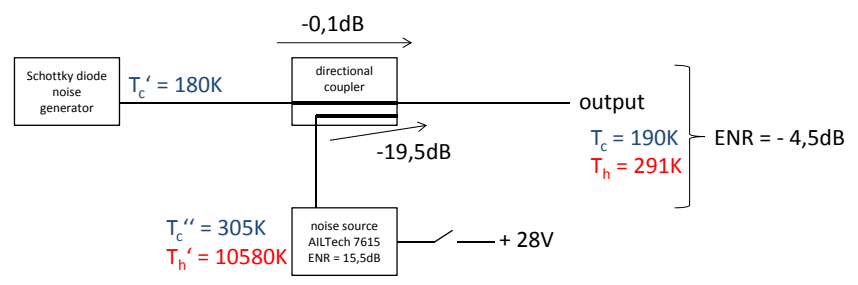

Fig. 8. Combining Schottky noise generator and standard noise source for "new" noise source with decreased overall cold temperature.

surements taken with an HP346A noise source (NF measured $0.97 \mathrm{~dB})$.

\section{Conclusions}

In this paper a broadband "cold" matched noise source providing noise temperatures below room temperature based on a Schottky diode was described. Its application in a noise figure measurement setup with enhanced accuracy was shown. Because of the decreased cold temperature in comparison to the usually used noise sources a lower hot temperature provided by more attenuation and thus less gain error - see Bertelsmeier (1988) - can be applied in $Y$ factor measurement method. Another advantage of the proposed noise generator is the decreased tolerance in effective ENR value due to decreased cold temperature.

Future work will evaluate in more detail the noise temperature uncertainty of the Schottky noise generator and its impact on NF measurement uncertainty. 


\section{Appendix A}

Starting with the basic equation which is evaluated in a noise figure measurement setup we have (see Pozar, 2000; Bertelsmeier, 1988)

$F+\frac{\mathrm{ENR}-Y \frac{T_{\mathrm{c}}}{T_{\mathrm{o}}}-1}{Y-1}$

where

- F: noise figure of device under test - linear scale,

- ENR: excess noise ratio of noise source - linear scale,,

- $Y$ : power ratio of power at output of DUT when noise source switched on to power at output of DUT when noise source switched off - linear scale,

- $T_{\mathrm{c}}$ : cold noise temperature of noise source (switched off) in $\mathrm{K}$,

- $T_{\mathrm{O}}$ : reference noise temperature, $T_{\mathrm{O}}=290 \mathrm{~K}$.

Furthermore the relation between equivalent noise temperature $T_{\mathrm{e}}$ given in Kelvin of the DUT and its (linear) noise figure $F$ is given by (see e.g. Pozar, 2000)

$F=1+\frac{T_{\mathrm{e}}}{T_{\mathrm{o}}}$

For the noise figure of the DUT in $\mathrm{dB}$ it holds

$N F_{\mathrm{dB}}=10 \cdot \log (F)$

Furthermore the definition of the (linear) ENR is given by

$\mathrm{ENR}=\frac{T_{\mathrm{h}}-T_{\mathrm{c}}}{T_{\mathrm{O}}}$

where $T_{\mathrm{h}}$ is the hot temperature when the noise source is switched on. Mostly ENR is given in dB's, thus

$\mathrm{ENR}_{\mathrm{dB}}=10 \cdot \log \frac{T_{\mathrm{h}}-T_{\mathrm{c}}}{T_{\mathrm{o}}}=10 \cdot \log (\mathrm{ENR})$

The measured $Y$ factor could be expressed in terms of $T_{\mathrm{e}}, T_{\mathrm{h}}$ and $T_{\mathrm{c}}$ according to

$Y=\frac{T_{\mathrm{h}}+T_{\mathrm{e}}}{T_{\mathrm{c}}+T_{\mathrm{e}}}$

or in dB's

$Y_{\mathrm{dB}}=10 \cdot \log (Y)$

As can be seen from equation Eq. (A1) two major sources of error in a noise figure measurement setup are the ENR calibration uncertainty and $Y$ factor measurement uncertainty. For state of the art noise sources, e.g. a HP346A an
$\mathrm{ENR}=5 \ldots 6 \mathrm{~dB}$ and an uncertainty of $\Delta \mathrm{ENR}= \pm 0.2 \mathrm{~dB}$ are specified. The uncertainty of $Y$ factor measurement is specified in terms of instrument uncertainty, e.g. $\Delta Y= \pm 0.1 \mathrm{~dB}$ for an HP8970.

Obviously these two variables are independent and thus differential calculus in the form of Taylor series and combining the uncertainties in a root-sum-of-squares fashion can be applied to find the uncertainty of $F$ :

$\Delta F=\sqrt{\left(\frac{\delta F}{\delta Y} \cdot \Delta Y\right)^{2}+\left(\frac{\delta F}{\delta \mathrm{ENR}} \cdot \Delta \mathrm{ENR}\right)^{2}}$

The partial derivatives of the function given in Eq. (A1) to the respective variables needed in equation Eq. (A8) are given as follows:

$\frac{\delta F}{\delta Y}=-\frac{\mathrm{ENR}+1}{(Y-1)^{2}}+\frac{T_{\mathrm{c}}}{T_{\mathrm{o}}} \cdot \frac{1}{(Y-1)^{2}}$

and

$\frac{\delta F}{\delta \mathrm{ENR}}+\frac{1}{Y-1}$

with

$\Delta \mathrm{ENR}=10^{\frac{\mathrm{ENR}_{\mathrm{dB}}}{10}} \cdot\left(10^{\frac{\mathrm{ENR}_{\mathrm{dB}}}{10}}-1\right)$

and

$\Delta Y=Y \cdot\left(10^{\frac{\Delta Y_{\mathrm{dB}}}{10}}-1\right)$

The linear $\Delta \mathrm{F}$ according to Eq. (A8) can thus be calculated as a function of $\Delta \mathrm{ENR}, \Delta Y$ and $T_{\mathrm{c}}-$ or in terms of $\mathrm{dB}$ it holds

$\Delta N F_{\mathrm{dB}}=\frac{10}{\log (10)} \cdot \frac{\Delta F}{F}$

which is the resulting NF uncertainty.

\section{References}

Zhutyaew, S.: 1296 MHz Small EME Station with Good Capability (part 1-4), available at: www.vhfdx.ru, 2010.

Maas, S. A.: Microwave Mixers, Artech House, 23-27, 1993.

Schiek, B., Rolfes, I., and Siwers, H.-J.: Noise in High-Frequency Circuits and Oscillators, Wiley, 133-136, 2006.

Pozar, D.: Microwave and RF Design of Wireless Systems, Wiley, 87-97, 2000.

Bertelsmeier, R.: Low Noise GaAs-FET Preamps for EME: Construction and Measurement Problems, DUBUS 4, 1988.

Swain, H. L. and Cox, R. M.: Noise Figure Meter Sets Records for Accuracy, Repeatability, and Convenience, Hewlett-Packard J., 4, 23-24, 1983. 